\title{
BMJ Open Is preoperative physical activity related to post-surgery recovery? A cohort study of patients with breast cancer
}

\author{
Hanna Nilsson, ${ }^{1}$ Ulf Angerås, ${ }^{1}$ David Bock, ${ }^{1}$ Mats Börjesson, ${ }^{2}$ Aron Onerup, ${ }^{1}$ \\ Monika Fagevik Olsen, ${ }^{3}$ Martin Gellerstedt, ${ }^{1,4}$ Eva Haglind, ${ }^{1}$ Eva Angenete ${ }^{1}$
}

To cite: Nilsson $\mathrm{H}$, Angerås U, Bock D, et al. Is preoperative physical activity related to post-surgery recovery? A cohort study of patients with breast cancer. BMJ Open 2016;6:e007997. doi:10.1136/bmjopen-2015007997

- Prepublication history and additional material is available. To view please visit the journal (http://dx.doi.org/ 10.1136/bmjopen-2015007997).

Received 19 February 2015 Revised 7 November 2015 Accepted 9 November 2015

CrossMark

For numbered affiliations see end of article.

\section{Correspondence to} Dr Hanna Nilsson; hanna.nilsson@vgregion.se

\section{ABSTRACT}

Objective: The aim of our study is to assess the association between preoperative level of activity and recovery after breast cancer surgery measured as hospital stay, length of sick leave and self-assessed physical and mental recovery.

Design: A prospective cohort study.

Setting: Patients included were those scheduled to undergo breast cancer surgery, between February and November 2013, at two participating hospitals in the Western Region of Sweden.

Participants: Patients planned for breast cancer surgery filled out a questionnaire before, as well as at 3 and 6 weeks after the operation. The preoperative level of activity was self-assessed and categorised into four categories by the participants using the 4-level SaltinGrimby Physical Activity Level Scale (SGPALS).

Main outcome measure: Our main outcome was postoperative recovery measured as length of sick leave, in-hospital stay and self-assessed physical and mental recovery.

Results: 220 patients were included. Preoperatively, $14 \%(31 / 220)$ of participants assessed themselves to be physically inactive, $61 \%(135 / 220)$ to exert some light physical activity (PA) and 20\% (43/220) to be more active (level $3+4$ ). Patients operated with mastectomy versus partial mastectomy and axillary lymph node dissection versus sentinel node biopsy were less likely to have a short hospital stay, relative risk (RR) 0.88 (0.78 to 1.00$)$ and 0.82 (0.70 to 0.96$)$. More active participants (level 3 or 4 ) had an $85 \%$ increased chance of feeling physically recovered at 3 weeks after the operation, RR 1.85 (1.20 to 2.85). No difference was seen after 6 weeks.

Conclusions: The above study shows that a higher preoperative level of PA is associated with a faster physical recovery as reported by the patients 3 weeks post breast cancer surgery. After 6 weeks, most patients felt physically recovered, diminishing the association above. No difference was seen in length of sick leave or self-assessed mental recovery between inactive or more active patients.

\section{INTRODUCTION}

The level of physical activity (PA) is associated with the development and prognosis

\section{Strengths and limitations of this study}

- A prospective series of patients at two breast cancer units in Sweden. Few exclusion criteria and the short time period for inclusion leave little room for changes in clinical practice. The use of a validated question for assessment of physical activity is another strength.

- In observational studies such as this, associations are found and discussed. However, as with other observational studies, it is not possible to determine the exact cause and effect. There might be other important factors, left out from the analyses, that would otherwise have an effect on its result. Another weakness is that we do not have access to information regarding the tumour stage, which may be of importance for mental and physical recovery as well as hospital stay and time of sick leave.

of several chronic diseases. Physical inactivity is considered to be the fourth most important risk factor for overall death by the $\mathrm{WHO},{ }^{1}$ not least due to its effect on cardiovascular disease and its strong association with different forms of cancer, including colorectal and breast cancer. ${ }^{2}{ }^{3}$ Therefore, in the pursuit of increased health, increased PA in the population is of great importance.

Increased PA after breast cancer diagnosis has been associated with decreased breast cancer-specific and overall mortality. ${ }^{4}$ The length of postoperative recovery is of immense importance both for the patient's well-being and for the costs of society. In recent years, several attempts have been made to enhance recovery after surgical treatment. One well-known programme, dealing with recovery after colorectal surgery, ${ }^{6}$ includes early mobilisation as well as early nutrition and has been shown to reduce the length of hospital stay by 2.5 days and to significantly lower the rate of postoperative complications. ${ }^{7}$ Often measured in terms of hospital stay and sick leave, patients' 
own assessment of postoperative recovery may be added to widen the understanding of postoperative recovery. The lifestyle of a patient has been shown to have an impact on enhanced recovery. For example, smoking and alcohol abuse are known to reduce the speed of recovery. Postoperative PA, however, increases well-being during cancer treatment ${ }^{8}{ }^{9}$ and may have a potential role to enhance recovery. In patients undergoing treatment for breast cancer, increased PA has been reported to reduce fatigue, and to increase quality of life (QoL) and cardiorespiratory fitness. ${ }^{10-14}$ Enhancing an individual's functional capacity before surgery, that is, prehabilitation, has been reported to be beneficial in other areas of surgery such as colorectal cancer ${ }^{15}$ and thoracic surgery. ${ }^{16}$

However, little is known about the role of preoperative PA for the immediate postoperative recovery after breast cancer surgery. Thus, the aim of the present study is to assess the association of preoperative PA with postoperative recovery. The primary outcome measure is length of hospital stay. Secondary outcome measures are length of sick leave, and self-assessed mental and physical recovery at 3 and 6 weeks after surgery, in patients operated because of breast cancer.

\section{Patients and method}

Patients scheduled to undergo breast cancer surgery, between February and November 2013, at two participating hospitals in the Western Region of Sweden, were asked to answer questionnaires before the operation as well as at 3 and 6 weeks after the operation. The exclusion criteria in the cohort study were no signed informed consent as well as the inability to understand written and spoken information. Figure 1 presents the flow of patients in the study. In total 257 patients were asked to participate in the study; 37 were excluded due to exclusion criteria and 220 patients continued to answer the first questionnaire. The questionnaires were

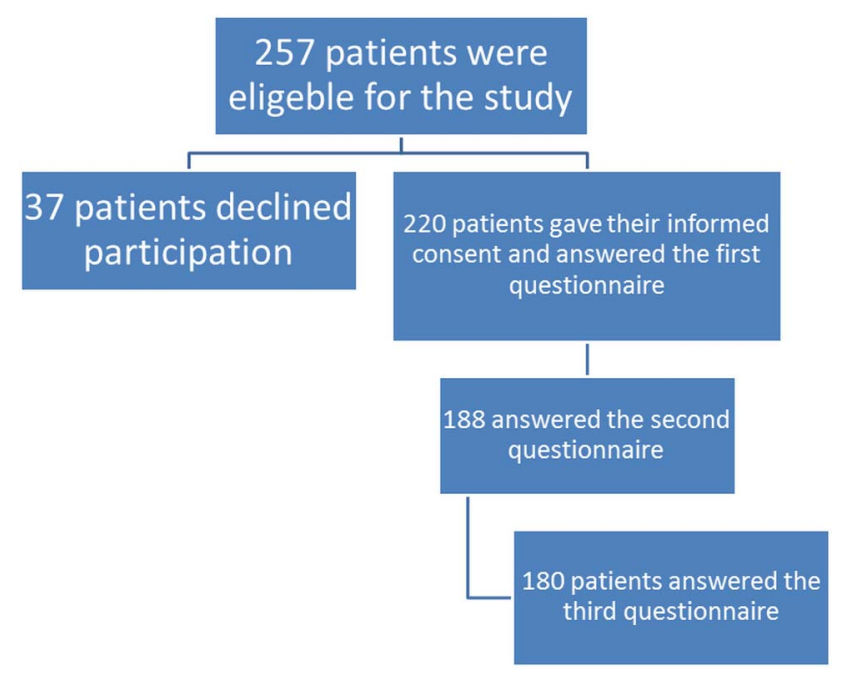

Figure 1 Flow chart of patients eligible for the study. designed by an expert panel consisting of surgeons, cardiologists with a special interest in PA, statisticians and nurses specialised in surgery, using well-validated methods. ${ }^{17}$ Thereafter, the questionnaire was validated face to face by patients undergoing breast cancer surgery using validation methods described previously. ${ }^{17} 18$

\section{Preoperative information}

Information concerning possible factors that may affect postoperative rehabilitation was retrieved from the preoperative questionnaire, including age, body mass index (BMI), marital status, comorbidity (hypertension, diabetes, hyperlipidaemia), depression, preoperative pain, general QoL, as well as smoking (yes/no) and alcohol habits. Moderate/heavy consumption for alcohol was set at 1 unit at least once per week during the last month. QoL was measured using the validated instrument EQ5D visual analogue scale $(0-100),{ }^{13} 19$ and general QoL, physical health and mental well-being were assessed in an ordinal seven-point Likert-type response format. ${ }^{20}$ The patients indicated one of seven numbers on a line anchored by, for example, 'no mental wellbeing' and 'the best possible mental well-being'. The answers 0-6 were dichotomised into 0-2 and 3-6. Depression was evaluated with one single question with the answer options 'yes', 'no' and 'I don't know'. This question has previously been found to correlate well with established depression scales. ${ }^{21}$

\section{Physical activity}

PA was assessed using the self-reported four-level Saltin-Grimby Physical Activity Level Scale (SGPALS), ${ }^{22}$ where patients answered the following question referring to the past week: 'How much do you move and exert yourself physically during leisure time? If your activity varies greatly try to estimate an average'. The answering categories were:

1. Physically inactive: Being almost completely inactive, reading, watching television, watching movies, using computers or doing other sedentary activities during leisure time.

2. Some light PA: Being physically active for at least $4 \mathrm{~h} /$ week, for example, riding a bicycle or walking to work, walking with the family, gardening, fishing, table tennis, bowling, etc.

3. Regular PA and training: Spending time on heavy gardening, running, swimming, playing tennis, badminton, callisthenics and similar activities for at least 2-3 h/week.

4. Regular hard physical training for competition sports: Spending time in running, orienteering, skiing, swimming, soccer, European handball, etc several times per week.

In our cohort, only one patient considered her activity to be regular, hard physical training, and therefore groups 3 and 4 were merged into one in the analyses below, as has been described previously. ${ }^{23}$ SGPALS has 
been shown to have a high validity and reliability, being associated to cardiovascular risk factors, ${ }^{24}{ }^{25}$ morbidity as well as mortality. ${ }^{23} 26$

\section{Perioperative information}

Information regarding type of operation, associated surgery and metastasis in axillary lymph node or elsewhere was retrieved from hospital administration systems using surgical codes and International Classification of Diseases (ICD) 10 diagnoses. Surgery was divided into partial mastectomy versus mastectomy and associated surgery into axillary lymph node dissection versus sentinel lymph node biopsy/no axillary surgery. ICD diagnosis C773 was used to identify patients with disseminated disease.

\section{Postoperative recovery}

Three and 6 weeks after the operation, a secondary questionnaire regarding postoperative rehabilitation was sent to the patients after a preceding phone call by a research nurse. Postoperative recovery was measured as hospital stay (days), length of sick leave (weeks) and the degree of postoperative physical and mental recovery.

Length of sick leave was measured in weeks, derived from the information given by the patient in the questionnaire. In the analyses, a cut-off was set after 3 weeks of sick leave, according to guidelines from the National Board of Health and Welfare ${ }^{27}$ outlined for patients operated by sector resection.

Length of hospital stay was retrieved using the hospital record and calculated in days, from the day of operation until the day the patient left the hospital.

Postoperative physical and mental recovery was self-assessed using the following question: 'To what extent do you feel physically/mentally fully recovered?' Answering categories were:

1. Not at all;

2. I feel recovered up to $25 \%$;

3. I feel recovered up to $50 \%$;

4. I feel recovered up to $75 \%$;

5. I feel completely recovered.

The answers were dichotomised into highly recovered (75-100\%) and incomplete recovery $(0-50 \%)$ for physical and mental recovery, respectively. For the outcome analyses, the relative chance of being highly recovered was calculated at 3 weeks.

\section{Statistical analysis}

All data were collected in a database and statistical analysis was performed using SAS V.9.3 software (SAS Institute). Patient characteristics were summarised descriptively. To assess the objective of the study, a Poisson regression model with a robust error variance and with PA as a fixed effect was used. ${ }^{28}$ Variables deemed to influence postoperative recovery were prespecified as age, type of surgery, smoking status, alcohol consumption and marital status. These variables were adjusted for in the model by including them as additional factors in a multivariable model. Results were reported as relative risk, 95\% CIs and $\mathrm{p}$ values from Wald tests. No correction for multiple testing was made and results should therefore be regarded as an interesting finding rather than as conclusive evidence. Loss to follow-up was addressed with a dropout analysis, aiming at finding the difference in baseline characteristics between participants who continued in the study and those lost to follow-up.

Permission to conduct the study was obtained from the Regional Ethics Board in Gothenburg, 2012-04-05 with DNR 180-12. The protocol was registered at clinicaltrials.gov 2013-12-12, with Clinicaltrials.gov identifier NCT01707121.

\section{RESULTS}

In total, 220 patients, 219 women and 1 man, were included in the study and answered the first preoperative questionnaire. Eighty-five per cent (188 patients) answered the second questionnaire after 3 weeks and 82\% (180 patients) answered all three questionnaires. Baseline patient and operative characteristics are seen in table 1. Output characteristics versus level of PA are to be found in online supplementary material.

Fourteen per cent $(31 / 220)$ of the patients assessed themselves as physically inactive at baseline and $61 \%$ $(135 / 220)$ as exerting some light PA. One-fifth $(43 / 220)$ rated themselves as more active (level $3+4$ ), while $5 \%$ $(11 / 220)$ did not answer the question. Inactive patients at baseline had a significantly higher BMI, a higher proportion of comorbidities, a lower QoL, as well as a lower mental well-being. In the dropout analysis, no difference in baseline characteristics, as outlined in table 1, was seen between patients who continued in the study and those who dropped out before week $3(n=40)$.

\section{Hospital stay and length of sick leave}

The relative chance of having a hospital stay for $<2$ days is listed in table 2 as relative risk (RR). The majority of patients, $91 \%$ (186/205), stayed $0-1$ day at the hospital while 19 patients stayed beyond the first day. Four patients, all operated with mastectomy, were in need of a reoperation due to bleeding. They had a longer hospital stay of $4,5,5$ and 9 days, respectively. Patients subjected to a more extensive surgery such as mastectomy and axillary lymph node dissection had a higher risk for a long hospital stay compared to patients who underwent partial mastectomy, RR for a short hospital stay 0.88 ( 0.78 to 1.0$)$ and sentinel lymph node dissection, RR for a short hospital stay 0.82 (0.70 to 0.96$)$. Smokers and participants with a moderate/higher alcohol consumption had a shorter hospital stay compared to nonsmokers and patients with a low alcohol consumption, RR 1.08 (1.00 to 1.16 ) and 1.10 (1.01 to 1.19 ).

Fifty-two patients $(43 \%)$, aged 66 years or less, were on sick leave for more than 3 weeks. The preoperative level of PA did not have any significant association with time of 
Table 1 Characteristics of patients versus preoperative level of physical activity

\begin{tabular}{|c|c|c|c|c|c|}
\hline & \multicolumn{3}{|c|}{ Level of physical activity* } & \multirow[b]{2}{*}{ Not reported } & \multirow[b]{2}{*}{ Total } \\
\hline & 1 & 2 & $3-4$ & & \\
\hline Patients, \% (N) & $14(31)$ & $61(135)$ & $20(43)$ & $5(11)$ & $100(220)$ \\
\hline Age, mean, years (SD) & $61.8(14.7)$ & $61.0(11.2)$ & $56.8(11.4)$ & $66.1(14.7)$ & $60.5(11.9)$ \\
\hline \multicolumn{6}{|l|}{ Social status, \% (n) } \\
\hline Married/cohabiting & $65(20)$ & $71(96)$ & $72(31)$ & $82(9)$ & $71(156)$ \\
\hline BMI, mean (SD), \% (n) & $28.4(5.4)$ & $25.9(4.5)$ & $24.7(3.6)$ & $29.9(5.9)$ & $26.2(4.7)$ \\
\hline Obesity $(>30)$ & $32(10)$ & $16(21)$ & $16(7)$ & $27(3)$ & $19(41)$ \\
\hline \multicolumn{6}{|l|}{ Comorbidity, $\uparrow \%$ (n) } \\
\hline Yes & $45(14)$ & $30(41)$ & $19(8)$ & $64(7)$ & $32(70)$ \\
\hline \multicolumn{6}{|l|}{ Smoking, \% (n) } \\
\hline Yes & $16(5)$ & $6(8)$ & $12(5)$ & & $8(18)$ \\
\hline \multicolumn{6}{|l|}{ Alcohol intake, \% (n) } \\
\hline Moderate/heavy consumption $\ddagger$ & $39(12)$ & $44(59)$ & $56(24)$ & $64(7)$ & $46(102)$ \\
\hline \multicolumn{6}{|l|}{ Preoperative pain, $\%(n)$} \\
\hline Intermediate/severe & $62(19)$ & $50(68)$ & $37(16)$ & $45(5)$ & 49 (107) \\
\hline \multicolumn{6}{|l|}{ Anxiety, \% (n) } \\
\hline Intermediate/severe & $52(16)$ & $49(66)$ & $51(22)$ & $27(3)$ & 49 (107) \\
\hline \multicolumn{6}{|l|}{ Method of operation, \% (n) } \\
\hline Mastectomy & $29(9)$ & $32(43)$ & $12(5)$ & $45(5)$ & $28(62)$ \\
\hline Sector & $58(18)$ & $61(83)$ & $84(36)$ & $55(6)$ & $65(143)$ \\
\hline \multicolumn{6}{|l|}{ Associated surgery, \% (n) } \\
\hline Sentinel node/no axillary operation & $77(24)$ & $80(108)$ & $72(31)$ & $73(8)$ & $80(171)$ \\
\hline Axillary lymph node dissection & $23(7)$ & $19(26)$ & $28(12)$ & $27(3)$ & $20(48)$ \\
\hline \multicolumn{6}{|l|}{ Disseminated disease, $\%(n)$} \\
\hline No diagnosis of disseminated disease & $84(26)$ & $80(108)$ & $74(32)$ & $82(9)$ & $80(175)$ \\
\hline Disseminated disease (ICD-10-C773) & $16(5)$ & $19(26)$ & $26(11)$ & $18(2)$ & $20(44)$ \\
\hline \multicolumn{6}{|l|}{ Quality of life, $\S \%(n)$} \\
\hline $0-2$ & $23(7)$ & $13(18)$ & $12(5)$ & $18(2)$ & $15(32)$ \\
\hline $3-6$ & $77(24)$ & $87(117)$ & $88(38)$ & $82(9)$ & $85(188)$ \\
\hline \multicolumn{6}{|l|}{ Mental well-being,§ \% (n) } \\
\hline $0-2$ & $35(11)$ & $8(11)$ & $12(5)$ & $27(3)$ & $14(30)$ \\
\hline $3-6$ & $65(20)$ & $92(124)$ & $88(38)$ & $73(8)$ & $86(190)$ \\
\hline \multicolumn{6}{|l|}{ Physical health,§ \% (n) } \\
\hline $0-2$ & $16(5)$ & $18(25)$ & $28(12)$ & $18(2)$ & $20(44)$ \\
\hline $3-6$ & $84(26)$ & 81 (109) & $72(31)$ & $82(9)$ & $80(175)$ \\
\hline EQ5D, VAS, mean (SD) & $61.2(19.8)$ & 74.9 (17.2) & $75.8(21.3)$ & 40 & $73.0(19.1)$ \\
\hline \multicolumn{6}{|l|}{ Hospital stay } \\
\hline 0-1 day & $71(22)$ & $85(115)$ & $93(40)$ & $82(9)$ & $85(186)$ \\
\hline 2 days or more & $13(4)$ & $9(12)$ & $2(1)$ & $18(2)$ & 9 (19) \\
\hline
\end{tabular}

sick leave, and nor did any of the other lifestyle factors included in the multivariate analysis shown in table 3.

\section{Self-assessed physical and mental recovery}

The relative chances of feeling physically or mentally recovered, at 3 weeks postoperatively, are listed in tables 4 and 5 as relative risk. Patients who assessed their preoperative PA to be higher; that is, grade 3 or 4, had an $85 \%$ increased chance of feeling physically recovered at 3 weeks after the operation, RR 1.85 (1.20 to 2.85). The association was not sustained after 6 weeks, 1.17 (0.92 to
1.49). Patients operated with an axillary lymph node dissection had a smaller chance of feeling physically recovered at 3 weeks than patients undergoing sentinel lymph node biopsy or none at all, 0.74 (0.57 to 0.97 ).

The level of PA did not matter significantly for mental recovery, whereas marital status did, as shown in table 5 . Participants in a relationship but living alone had a decreased chance of feeling mentally recovered compared to participants who were married or cohabiting, RR 0.77 (0.61 to 0.97). The effect was sustained after 6 weeks, 0.80 (0.68 to 0.95$)$. 
Table 2 Relative chance of having a hospital stay $\leq 1$ day

\begin{tabular}{|c|c|c|c|c|c|}
\hline & \multirow{2}{*}{$\begin{array}{l}\text { Number of } \\
\text { patients included }\end{array}$} & \multicolumn{2}{|l|}{ Univariate } & \multicolumn{2}{|l|}{ Multivariate } \\
\hline & & $\mathbf{R R}$ & p Value & $\mathbf{R R}$ & p Value \\
\hline \multicolumn{6}{|l|}{ Marital status } \\
\hline Married/cohabiting & 136 & Ref & & Ref & \\
\hline Living alone & 43 & 1.01 (0.90 to 1.12$)$ & 0.924 & 0.97 (0.88 to 1.08$)$ & 0.596 \\
\hline Living apart & 13 & $0.91(0.87$ to 0.96$)$ & 0.001 & 0.94 (0.86 to 1.02$)$ & 0.120 \\
\hline \multicolumn{6}{|l|}{ Smoking } \\
\hline No & 173 & Ref & & Ref & \\
\hline Yes & 18 & $1.10(1.05$ to 1.16$)$ & 0.001 & 1.08 (1.00 to 1.16$)$ & 0.043 \\
\hline \multicolumn{6}{|l|}{ Method of surgery } \\
\hline Partial mastectomy & 135 & Ref & & Ref & \\
\hline Mastectomy & 57 & 0.80 (0.69 to 0.92$)$ & 0.002 & 0.88 (0.78 to 1.00$)$ & 0.037 \\
\hline \multicolumn{6}{|l|}{ Associated surgery } \\
\hline Sentinel node/no axillary operation & 149 & Ref & & Ref & \\
\hline Axillary lymph node dissection & 45 & 0.78 (0.67 to 0.93$)$ & 0.004 & $0.82(0.70$ to 0.96$)$ & 0.013 \\
\hline Age & 190 & $-0.00(-0.01$ to 0.00$)$ & 0.732 & $-0.00(-0.00$ to 0.00$)$ & 0.933 \\
\hline \multicolumn{6}{|l|}{ Alcohol intake } \\
\hline Modest & 104 & Ref & & Ref & \\
\hline Moderate/heavy* & 88 & 1.12 (1.02 to 1.22$)$ & 0.012 & $1.10(1.01$ to 1.19$)$ & 0.026 \\
\hline \multicolumn{6}{|l|}{ Saltin-Grimby Activity Level Scale† } \\
\hline 1 & 26 & Ref & & & \\
\hline 2 & 127 & 1.07 (0.90 to 1.27$)$ & 0.443 & 1.09 (0.93 to 1.29$)$ & 0.315 \\
\hline $3+4$ & 41 & $1.15(0.97$ to 1.37$)$ & 0.103 & $1.13(0.98$ to 1.32$)$ & 0.051 \\
\hline
\end{tabular}

\section{Follow-up at 6 weeks}

After 6 weeks, no differences were seen between the analysed lifestyle factors for sick leave, nor for physical recovery.

\section{DISCUSSION}

The main finding of this study is that the preoperative PA level is positively associated with an enhanced physical recovery after breast cancer surgery. More extensive surgery was associated with a longer hospital stay and a smaller chance of feeling physically recovered after 3 weeks. After 6 weeks, this difference is reduced to nonsignificance level since most patients, independent of the type of operation, preoperative PA level or other lifestyle factor studied, consider themselves to be recovered. Being in a relationship, but living alone was associated with slower mental recovery postoperatively compared to those being married or cohabiting. However, our primary end point sick leave was not affected by any of the studied lifestyle factors, including PA.

\section{Strength and weaknesses of the study}

The strength of this study is that it is a prospective series of patients at two breast cancer units in Sweden. Few exclusion criteria and the short time period for inclusion leave little room for changes in clinical practice. Another strength is the use of a validated question for assessment of PA. This single question, SGPALS, is easy to use in clinical practice and has been validated against cardiovascular risk factors, morbidity and mortality. Rödjer $e t a l^{23}$ showed that SGPALS correlated to waist circumference, resting heart rate and levels of plasma glucose, serum triglycerides and low-density lipoproteins. Of 1903 women, randomly chosen and living in the western part of Sweden, $9 \%$ of women considered themselves to be inactive, $66 \%$ exerted some light PA and $25 \%$ perceived themselves to be moderately or vigorously physically active, ${ }^{23}$ proportions well correlated to what we found in our study and therefore indicating that the 119 women included in our study may well be representative of the normal population selected only for surgery.

In observational studies such as this, associations are found and discussed. However, as with other observational studies, it is not possible to determine the exact cause and effect. For example, PA measured by SGPALS has also been found to correlate well with stress and mental health. In our study we did not determine mental health, and therefore it is not possible to differentiate between the direct effect of a high level of PA and god mental health as possible causes for enhanced recovery. There might be other important factors, left out from the analyses, that would otherwise have had an effect on its result. 
Table 3 Relative chance of sick leave shorter than 3 weeks

\begin{tabular}{|c|c|c|c|c|c|}
\hline & \multirow{2}{*}{$\begin{array}{l}\text { Number of } \\
\text { patients included }\end{array}$} & \multicolumn{2}{|l|}{ Univariate } & \multicolumn{2}{|l|}{ Multivariate } \\
\hline & & RR & p Value & $\mathbf{R R}$ & p Value \\
\hline \multicolumn{6}{|l|}{ Marital status } \\
\hline Married/cohabiting & 75 & Ref & & Ref & \\
\hline Living alone & 16 & $1.16(0.64$ to 2.11$)$ & 0.631 & 1.29 (0.69 to 2.42$)$ & 0.423 \\
\hline Living apart & 9 & 0.91 (0.49 to 1.70$)$ & 0.773 & 0.79 (0.42 to 1.47$)$ & 0.454 \\
\hline \multicolumn{6}{|l|}{ Smoking } \\
\hline No & 94 & Ref & & Ref & \\
\hline Yes & 5 & $0.83(0.40$ to 1.75$)$ & 0.592 & 1.37 (0.68 to 2.75$)$ & 0.374 \\
\hline \multicolumn{6}{|l|}{ Method of surgery } \\
\hline Partial mastectomy & 71 & Ref & & Ref & \\
\hline Mastectomy & 26 & 1.61 (0.94 to 2.74$)$ & 0.082 & $1.42(0.78$ to 2.60$)$ & 0.254 \\
\hline \multicolumn{6}{|l|}{ Associated surgery } \\
\hline Sentinel node/no axillary operation & 79 & Ref & & Ref & \\
\hline Axillary lymph node dissection & 21 & 0.50 (0.25 to 0.99$)$ & 0.046 & $0.52(0.265$ to 1.02$)$ & 0.058 \\
\hline Age & 98 & $0.00(-0.02$ to 0.03$)$ & 0.723 & $0.00(-0.02$ to 0.02$)$ & 0.913 \\
\hline \multicolumn{6}{|l|}{ Alcohol intake } \\
\hline Modest & 51 & Ref & & Ref & \\
\hline Moderate/heavy* & 49 & 0.89 (0.60 to 1.32 & 0.550 & 0.78 (0.50 to 1.22$)$ & 0.275 \\
\hline \multicolumn{6}{|l|}{ Saltin-Grimby Activity Level Scale† } \\
\hline 1 & 10 & Ref & & Ref & \\
\hline 2 & 64 & 0.94 (0.48 to 1.84$)$ & 0.851 & 1.10 (1.56 to 2.17$)$ & 0.783 \\
\hline $3+4$ & 26 & 1.15 (0.57 to 2.32$)$ & 0.689 & $1.42(0.71$ to 2.84$)$ & 0.320 \\
\hline
\end{tabular}

Table 4 Relative chance of feeling physically recovered 3 weeks after the operation

\begin{tabular}{|c|c|c|c|c|c|}
\hline & \multirow{2}{*}{$\begin{array}{l}\text { Number of } \\
\text { patients included }\end{array}$} & \multicolumn{2}{|l|}{ Univariate } & \multicolumn{2}{|l|}{ Multivariate } \\
\hline & & $\mathbf{R R}$ & p Value & $\mathbf{R R}$ & p Value \\
\hline \multicolumn{6}{|l|}{ Marital status } \\
\hline Married/cohabiting & 122 & Ref & & Ref & \\
\hline Living alone & 35 & $0.90(0.77$ to 1.10$)$ & 0.365 & $0.93(0.79$ to 1.11$)$ & 0.424 \\
\hline Living apart & 13 & $1.01(0.74$ to 1.40$)$ & 0.950 & 0.95 (0.72 to 1.24$)$ & 0.683 \\
\hline \multicolumn{6}{|l|}{ Smoking } \\
\hline No & 153 & Ref & & Ref & \\
\hline Yes & 15 & $1.00(0.76$ to 1.30$)$ & 0.971 & 1.10 (0.88 to 1.37$)$ & 0.392 \\
\hline \multicolumn{6}{|l|}{ Method of surgery } \\
\hline Partial mastectomy & 113 & Ref & & Ref & \\
\hline Mastectomy & 50 & 0.77 (0.62 to 0.95$)$ & 0.015 & $0.92(0.75$ to 1.13$)$ & 0.434 \\
\hline \multicolumn{6}{|l|}{ Associated surgery } \\
\hline Sentinel node/no axillary operation & 129 & Ref & & Ref & \\
\hline Axillary lymph node dissection & 41 & 0.73 (0.58 to 0.93$)$ & 0.010 & $0.74(0.57$ to 0.97$)$ & 0.027 \\
\hline Age & 167 & $-0.00(-0.00$ to 0.00$)$ & 0.390 & $-0.00(-0.01$ to 0.00$)$ & 0.582 \\
\hline \multicolumn{6}{|l|}{ Alcohol intake } \\
\hline Modest & 92 & Ref & & Ref & \\
\hline Moderate/heavy* & 77 & $0.96(0.82$ to 1.11$)$ & 0.565 & $0.90(0.76$ to 1.06$)$ & 0.196 \\
\hline \multicolumn{6}{|l|}{ Saltin-Grimby Activity Level Scale† } \\
\hline 1 & 22 & Ref & & Ref & \\
\hline 2 & 114 & 1.35 (0.94 to 1.94$)$ & 0.101 & 1.49 (0.96 to 2.30$)$ & 0.074 \\
\hline $3+4$ & 35 & $1.60(1.12$ to 2.28$)$ & 0.010 & $1.85(1.20$ to 2.85$)$ & 0.006 \\
\hline
\end{tabular}


Table 5 Relative chance of feeling mentally recovered 3 weeks after the operation

\begin{tabular}{|c|c|c|c|c|c|}
\hline & \multirow{2}{*}{$\begin{array}{l}\text { Number of } \\
\text { patients included }\end{array}$} & \multicolumn{2}{|l|}{ Univariate } & \multicolumn{2}{|l|}{ Multivariate } \\
\hline & & RR & p Value & RR & p Value \\
\hline \multicolumn{6}{|l|}{ Marital status } \\
\hline Married/cohabiting & 122 & Ref & & Ref & \\
\hline Living alone & 33 & $0.85(0.71$ to 1.02$)$ & 0.080 & 0.84 ( 0.68 to 1.04$)$ & 0.121 \\
\hline Living apart & 13 & 0.85 (0.66 to 1.10$)$ & 0.223 & 0.77 (0.61 to 0.97$)$ & 0.028 \\
\hline \multicolumn{6}{|l|}{ Smoking } \\
\hline No & 152 & Ref & & Ref & \\
\hline Yes & 15 & $0.78(0.51$ to 1.19$)$ & 0.248 & $0.81(0.54$ to 1.23$)$ & 0.323 \\
\hline \multicolumn{6}{|l|}{ Method of surgery } \\
\hline Partial mastectomy & 113 & Ref & & Ref & \\
\hline Mastectomy & 48 & $1.00(0.82$ to 1.21$)$ & 0.976 & $1.10(0.89$ to 1.35$)$ & 0.389 \\
\hline \multicolumn{6}{|l|}{ Associated surgery } \\
\hline Sentinel node/no axillary operation & 128 & Ref & & Ref & \\
\hline Axillary lymph node dissection & 40 & $0.90(0.72$ to 1.12$)$ & 0.345 & $0.83(0.64$ to 1.09$)$ & 0.178 \\
\hline Age & 165 & $0.00(-0.01$ to 0.01$)$ & 0.710 & $0.00(-0.01$ to 0.01$)$ & 0.671 \\
\hline \multicolumn{6}{|l|}{ Alcohol intake } \\
\hline Modest & 91 & Ref & & Ref & \\
\hline Moderate/heavy* & 76 & $1.02(0.86$ to 1.20$)$ & 0.848 & $1.01(0.83$ to 1.23$)$ & 0.918 \\
\hline \multicolumn{6}{|l|}{ Saltin-Grimby Activity Level Scale† } \\
\hline 1 & 22 & Ref & & Ref & \\
\hline 2 & 113 & $1.21(0.87$ to 1.69$)$ & 0.260 & $1.23(0.82$ to 1.83$)$ & 0.314 \\
\hline $3+4$ & 34 & 1.25 (0.87 to 1.78$)$ & 0.227 & 1.38 (0.90 to 2.13$)$ & 0.144 \\
\hline
\end{tabular}

A limitation of our study is that there were eligible patients who were not included since the study nurse was not available at all hours. Another weakness is that we do not have access to information regarding tumour stage nor planned adjuvant therapy. However, patients in the cohort studied were given the response of their pathological examination 4 weeks after surgery when adjuvant therapy was decided on. Therefore, the initial hospital stay, initial sick leave and physical and mental recovery ought not to be affected by this.

\section{Interpretation of study}

This study adds to the evidence in favour of PA in patients undergoing treatment for breast cancer, showing an enhanced recovery in patients who preoperatively were more active. This is in line with earlier studies where increased PA in women treated for breast cancer is associated with an increase in QoL with longterm effect, ${ }^{29}$ decreased fatigue, and reduced breast cancer mortality as well as overall mortality. Most evidence arises from cohort trials, while there are also randomised controlled trials investigating the role of postoperative PA. ${ }^{14} 3031$ This study indicates that preoperative PA may also be of importance for recuperating from surgery, and hence for the patient to be able to initiate PA earlier.

Our aim was to investigate self-assessed recovery, mental and physical. After 6 weeks, the association between physical recovery and any of the lifestyle variables, including PA, had diminished into nonsignificant levels. This is probably explained by the fact that most patients felt completely recovered after 6 weeks, reflecting the mild physical trauma caused by breast cancer surgery. However, as stated above, studies with other end points such as quality of life ${ }^{32}$ show a positive effect of increased PA. For self-assessed mental recovery, marital status seemed to have a stronger association than other lifestyle factors, emphasising the importance of one's social network when mentally coping with a malignant disease.

In this study, it was not possible to determine any factor influencing our primary end point: the time of sick leave. In Sweden, the entire working population is covered by national sick leave insurance. The rationale of choosing 3 weeks is due to the guidelines set by the National Board of Health and Welfare for breast cancer treatment where they advised patients treated with partial mastectomy to be on sick leave for approximately 3 weeks. In our study, $66 \%$ of patients were operated with partial mastectomy. Possibly, this cut-off was too short since one-third of patients were subject to enlarged surgery, with a recommendation of a longer time of sick leave. Also, the homogeneous time of sick leave found in our study may be the result of patients being offered sick leave at discharge with a given standard.

Women subjected to axillary lymph node dissection had a longer hospital stay and felt physically recovered 
to a lesser extent, which is in line with the current knowledge of the side effects of this operation. Smokers and patients with a higher level of alcohol consumption left the hospital earlier than non-smokers and patients with a modest alcohol consumption, possibly reflecting that these habits are not easily satisfied inside hospitals in Sweden.

The association of PA with physical recovery, shown in this study, is clinically relevant. The SGPALS offers the clinician important information, and may in the future be used to target patients in need of specific intervention. The results of this study add to the current knowledge of the health benefits of PA and can be used as an additional argument for increasing the level of PA in insufficiently active populations. In addition to the current knowledge that PA reduces the risk of cancer as well as the mortality after breast cancer treatment, this study indicates that increased PA may influence postoperative recovery.

Indeed, the above study shows that patients with an increased level of PA assess their physical recovery to be faster. Whether or not it is meaningful to increase PA before breast cancer surgery (prehabilitation) remains to be studied. However, a recent study showed that prehabilitation managed to cause positive changes in postoperative functional exercise capacity after colorectal surgery. ${ }^{15}$ To the best of our knowledge, this is the first study of sick leave/return to work after breast cancer surgery in relation to the preoperative level of PA. In orthopaedic surgery, prehabilitation has been analysed in relation to return to work and suggested to be advantageous. ${ }^{33}$

In conclusion, being physically active has many positive effects for patients treated for breast cancer. This study adds to this knowledge by showing that a higher preoperative level of PA is associated with faster physical recovery. These findings have potential clinical implications, but need to be confirmed and expanded on in randomised controlled trials of preoperative PA interventions.

\section{Author affiliations \\ ${ }^{1}$ Department of Surgery, Institute of Clinical Sciences, Sahlgrenska Academy, University of Gothenburg, SSORG-Scandinavian Surgical Outcomes Research Group, Sahlgrenska University Hospital/Östra, Gothenburg, Sweden ${ }^{2}$ Swedish School of Sport and Health Sciences and Karolinska University Hospital, Stockholm, Sweden \\ ${ }^{3}$ Department of Gastrosurgical Research and Education, Gothenburg, Sweden \\ ${ }^{4}$ School of Business, Economics and IT, University West, Göteborg, Sweden}

Contributors UA, MB, MFO, MG, EA and EH have participated from the beginning by creating ideas for the study, designing the study, writing the study protocol, design of the questionnaire and finally applying to the regional ethical board. Together with MB, EA and EH, HN, DB and AO have contributed to the study in the process of data collection and data analyses. With the help of $\mathrm{AO}$, HN has written the manuscript and all the authors have contributed with ideas for the final manuscript. All the authors have read and accepted the final draft.

Funding The funding for the study has been raised from funds created by the Swedish Cancer Society, The Swedish Research Council and Region Västra Götaland, Sahlgrenska University Hospital (ALF grant 138751, agreement concerning research and education of doctors).
Competing interests None declared.

Ethics approval Regional Ethics Board in Gothenburg, 2012-04-05 with DNR 180-12. The protocol was registered at clinicaltrials.gov 2013-12-12, with Clinicaltrials.gov identifier NCT01707121.

Provenance and peer review Not commissioned; externally peer reviewed.

Data sharing statement No additional data are available.

Open Access This is an Open Access article distributed in accordance with the Creative Commons Attribution Non Commercial (CC BY-NC 4.0) license, which permits others to distribute, remix, adapt, build upon this work noncommercially, and license their derivative works on different terms, provided the original work is properly cited and the use is non-commercial. See: http:// creativecommons.org/licenses/by-nc/4.0/

\section{REFERENCES}

1. WHO. Physical activity, fact sheet $N^{\circ} 385.2014$. http://www.who.int/ mediacentre/factsheets/fs385/en/

2. Warburton DE, Nicol CW, Bredin SS. Health benefits of physical activity: the evidence. CMAJ 2006;174:801-9.

3. Friedenreich CM, Cust AE. Physical activity and breast cancer risk: impact of timing, type and dose of activity and population subgroup effects. Br J Sports Med 2008;42:636-47.

4. Ogunleye AA, Holmes MD. Physical activity and breast cancer survival. Breast Cancer Res 2009;11:106.

5. Holmes MD, Chen WY, Feskanich D, et al. Physical activity and survival after breast cancer diagnosis. JAMA 2005;293:2479-86.

6. Basse L, Hjort Jakobsen D, Billesbølle P, et al. A clinical pathway to accelerate recovery after colonic resection. Ann Surg 2000;232:51-7.

7. Varadhan $\mathrm{KK}, \mathrm{Neal} \mathrm{KR}$, Dejong $\mathrm{CH}$, et al. The enhanced recovery after surgery (ERAS) pathway for patients undergoing major elective open colorectal surgery: a meta-analysis of randomized controlled trials. Clin Nutr 2010;29:434-40.

8. Lee A, Chiu CH, Cho MW, et al. Factors associated with failure of enhanced recovery protocol in patients undergoing major hepatobiliary and pancreatic surgery: a retrospective cohort study. BMJ Open 2014;4:e005330.

9. Tonnesen $\mathrm{H}$, Rosenberg J, Nielsen $\mathrm{HJ}$, et al. Effect of preoperative abstinence on poor postoperative outcome in alcohol misusers: randomised controlled trial. BMJ 1999;318:1311-16.

10. McNeely ML, Campbell KL, Rowe BH, et al. Effects of exercise on breast cancer patients and survivors: a systematic review and meta-analysis. CMAJ 2006;175:34-41.

11. Knols R, Aaronson NK, Uebelhart D, et al. Physical exercise in cancer patients during and after medical treatment: a systematic review of randomized and controlled clinical trials. J Clin Oncol 2005;23:3830-42.

12. Schmitz KH, Holtzman J, Courneya KS, et al. Controlled physical activity trials in cancer survivors: a systematic review and meta-analysis. Cancer Epidemiol Biomarkers Prev 2005;14:1588-95.

13. EuroQol Group. EuroQol-a new facility for the measurement of health-related quality of life. Health Policy 1990;16:199-208.

14. Courneya KS, Mackey JR, Bell GJ, et al. Randomized controlled tria of exercise training in postmenopausal breast cancer survivors: cardiopulmonary and quality of life outcomes. J Clin Oncol 2003;21:1660-8.

15. Gillis $\mathrm{C}$, Li C, Lee $\mathrm{L}$, et al. Prehabilitation versus rehabilitation: a randomized control trial in patients undergoing colorectal resection for cancer. Anesthesiology 2014;121:937-47.

16. Hulzebos $\mathrm{EH}$, Helders PJ, Favié $\mathrm{NJ}$, et al. Preoperative intensive inspiratory muscle training to prevent postoperative pulmonary complications in high-risk patients undergoing CABG surgery: a randomized clinical trial. JAMA 2006;296:1851-7.

17. Steineck G, Helgesen F, Adolfsson J, et al. Quality of life after radical prostatectomy or watchful waiting. $N$ Engl J Med 2002;347:790-6.

18. Omerov $\mathrm{P}$, Steineck G, Runeson B, et al. Preparatory studies to a population-based survey of suicide-bereaved parents in Sweden. Crisis 2013;34:200-10.

19. Brooks R. EuroQol: the current state of play. Health Policy 1996;37:53-72.

20. Likert R. A technique for the measurement of attitudes. Arch Psychol 1932;22:55.

21. Skoogh J, Ylitalo N, Larsson Omeróv P, et al, Swedish-Norwegian Testicular Cancer Group. 'A no means no'-measuring depression using a single-item question versus Hospital Anxiety and Depression Scale (HADS-D). Ann Oncol 2010;21:1905-9. 
22. Saltin B, Grimby G. Physiological analysis of middle-aged and old former athletes. Comparison with still active athletes of the same ages. Circulation 1968;38:1104-15.

23. Rodjer L, Jonsdottir IH, Rosengren A, et al. Self-reported leisure time physical activity: a useful assessment tool in everyday health care. BMC Public Health 2012;12:693.

24. Thune I, Njølstad I, Løchen ML, et al. Physical activity improves the metabolic risk profiles in men and women: the Tromso Study. Arch Intern Med 1998;158:1633-40.

25. Wilhelmsen L, Tibblin G, Aurell M, et al. Physical activity, physical fitness and risk of myocardial infarction. Adv Cardiol 1976;18:217-30

26. Rosengren A, Wilhelmsen L. Physical activity protects against coronary death and deaths from all causes in middle-aged men. Evidence from a 20-year follow-up of the primary prevention study in Goteborg. Ann Epidemiol 1997;7:69-75.

27. Sjukskrivning vid bröstcancer, http://www.socialstyrelsen.se/riktlinjer/ forsakringsmedicinsktbeslutsstod/brostcancer-ickespridd. Editor. Helena Granstam Björneklett, Lotta Lundgren Eriksson, Carina Modéus, Christina Haapaniemi Ohlsson, Marie Sundquist, Anna-Karin Wennstig.
28. Zou G. A modified poisson regression approach to prospective studies with binary data. Am J Epidemiol 2004;159:702-6.

29. Courneya KS, Segal RJ, Gelmon K, et al. Six-month follow-up of patient-rated outcomes in a randomized controlled trial of exercise training during breast cancer chemotherapy. Cancer Epidemiol Biomarkers Prev 2007;16:2572-8.

30. Courneya KS, Friedenreich CM, Quinney HA, et al. A randomized trial of exercise and quality of life in colorectal cancer survivors. Eur $J$ Cancer Care (Engl) 2003;12:347-57.

31. Rogers LQ, Courneya KS, Anton PM, et al. Effects of the BEAT cancer physical activity behavior change intervention on physical activity, aerobic fitness, and quality of life in breast cancer survivors: a multicenter randomized controlled trial. Breast Cancer Res Treat 2015;149:109-19.

32. Schmidt T, Weisser B, Dürkop J, et al. Comparing endurance and resistance training with standard care during chemotherapy for patients with primary breast cancer. Anticancer Res 2015;35:5623-9.

33. Nielsen PR, Andreasen J, Asmussen M, et al. Costs and quality of life for prehabilitation and early rehabilitation after surgery of the lumbar spine. BMC Health Serv Res 2008;8:209. 